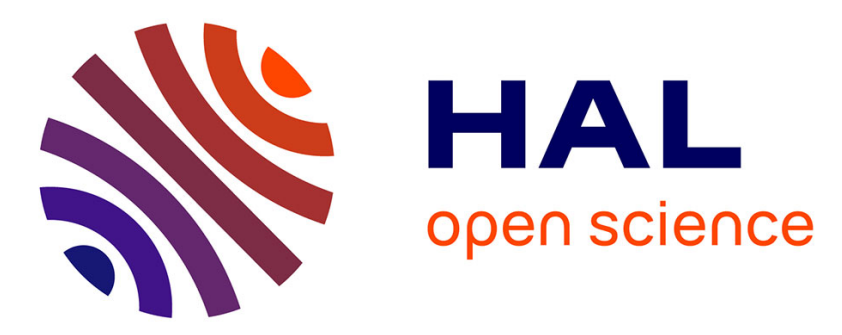

\title{
GATE, a Geant4-based simulation platform for PET integrating movement and time management
}

G. Santin, D. Strul, Delphine Lazaro, L. Simon, M. Krieguer, M. Vieira Martins, Vincent Breton, C. Morel

\section{- To cite this version:}

G. Santin, D. Strul, Delphine Lazaro, L. Simon, M. Krieguer, et al.. GATE, a Geant4-based simulation platform for PET integrating movement and time management. IEEE Nuclear Science Symposium and Medical Imaging Conference 2002 IEEE NSS/MIC, Nov 2002, Norfolk, United States. pp.1516-1521. in2p3-00012854

HAL Id: in2p3-00012854

https://hal.in2p3.fr/in2p3-00012854

Submitted on 11 Jun 2003

HAL is a multi-disciplinary open access archive for the deposit and dissemination of scientific research documents, whether they are published or not. The documents may come from teaching and research institutions in France or abroad, or from public or private research centers.
L'archive ouverte pluridisciplinaire HAL, est destinée au dépôt et à la diffusion de documents scientifiques de niveau recherche, publiés ou non, émanant des établissements d'enseignement et de recherche français ou étrangers, des laboratoires publics ou privés. 


\title{
GATE, a Geant4-based simulation platform for PET integrating movement and time management
}

\author{
G. Santin ${ }^{\mathrm{a}, \mathrm{b}}$, D. Strul ${ }^{\mathrm{a}, \mathrm{b}}$, D. Lazaro ${ }^{\mathrm{a}}$, L. Simon ${ }^{\mathrm{a}, \mathrm{b}}$, M. Krieguer ${ }^{\mathrm{a}, \mathrm{b}}$, M. Vieira Martins ${ }^{\mathrm{b}}$, V. Breton ${ }^{\mathrm{a}}$ and C. Morel ${ }^{\mathrm{a}, \mathrm{b}}$
}

\begin{abstract}
GATE, the Geant4 Application for Tomographic Emission, is a simulation platform developed for PET and SPECT. It combines a powerful simulation core (the Geant4 toolkit) and a large range of developments dedicated to nuclear medicine. In particular, it models the passing of time during real acquisitions, allowing to handle dynamic systems such as decaying source distributions or moving detectors. We present several series of results that illustrate the possibilities of this new platform. The simulation of decaying sources is illustrated on a dual-isotope acquisition with multiple time-frames. Count rate curves taking into account random coincidences and dead-time are shown for a dual-crystal set-up and for a small-animal PET scanner configuration. Simulated resolution curves and reconstructed images are shown for rotating PET scanners. Lastly, we present comparisons of simulated point-spread functions and spectra with experimental results obtained from a small-animal gamma camera prototype.
\end{abstract}

\section{INTRODUCTION}

A ccurate Monte-Carlo simulations are required for a large range of PET and SPECT applications that include scanner design, statistical image reconstruction, scatter correction and protocol optimization [1]. GATE, the Geant4 Application for Tomographic Emission, is an object-oriented simulation platform meant to provide a long-term, versatile solution for a large range of problems.

GATE is built on top of the multipurpose simulation toolkit Geant4 [2], and thus incorporates all Geant4 features, including well-validated physics models, geometry modeling tools, visualization and 3D rendering, and a scripting language for interactive command of the simulation. It integrates a large set of components dedicated to PET and SPECT, designed to

This work was supported by the Swiss National Foundation for Research under Grant No. 21-63870.00.

G. Santin was with the Institute of High Energy Physics, University of Lausanne, Switzerland. $\mathrm{He}$ is now with ESA-ESTEC, Noordwijk, The Netherlands (giovanni.santin@esa.int).

D. Strul, L. Simon and C. Morel are with the Institute of High Energy Physics, University of Lausanne, Switzerland (daniel.strul@iphe.unil.ch).

D. Lazaro and V. Breton are with the Laboratoire de Physique Corpusculaire, IN2P3 - CNRS, University of Clermont-Ferrand, France (lazaro@clermont.in2p3.fr).

M. Krieguer is with the Inter Institute of High Energy, Vrije Universiteit Brussel, Belgium (magalie.krieguer@iphe.unil.ch).

M. Vieira Martins is with the Institute of Biophysics and Biomedical Engineering, University of Lisbon, Portugal (d323@fc.ul.pt).

${ }^{a}$ Members of the OpenGATE Collaboration.

${ }^{\mathrm{b}}$ Members of the Crystal Clear Collaboration. allow the simulation of a wide range of acquisitions. Timedependence is taken into account at all steps of the simulation, so that realistic simulations of the acquisition count rates, or source decay can be achieved under dynamic configurations (e.g. rotating detectors, or evolving bio-distributions).

We describe hereafter the main characteristics of the GATE platform. We then present several series of results that illustrate the possibilities of this new platform. The simulation of decaying sources is illustrated on a dual-isotope acquisition with multiple time-frames. Count rate curves taking into account random coincidences and dead-time are shown for a dual-crystal set-up and for a small-animal PET scanner design. Simulated resolution curves and reconstructed images are shown for rotating PET scanners. Lastly, we present comparisons of simulated point-spread functions and spectra with experimental results obtained from a small-animal gamma camera prototype.

\section{DESCRIPTION OF THE MONTE-CARLO CODE}

GATE comprises of a large number of software components allowing to model various aspects of nuclear medicine experiments: geometry, movement, detection, data output, etc. Users can choose among these components and assemble them interactively to set up simulations according to their own requirements by using a scripting language.

One may specify any number of radioactive sources with different properties (radioisotope, position, activity), which may overlap on each other and may also overlap with the detector geometry (e.g. natural radioactivity of Lu). Users may interactively select which gamma-ray interactions should be considered among those provided by the Geant 4 low energy electromagnetic package (photoelectric effect, Compton and Rayleigh scattering, and gamma-ray conversion) and the energy cut applied to gamma-rays and electrons for their tracking. A number of modules is available for modeling the detection process, going from the detection of the gamma-rays by the scintillating crystals (singles) to the detection of coincidences. Scattered and random events are tagged and may be processed independently from true coincidences. Simulation data results may be stored on an event-by-event basis into multiple output files with different file formats (e.g. the Crystal Clear Collaboration's List Mode Format (LMF) for the small animal ClearPET scanner prototypes). 
a

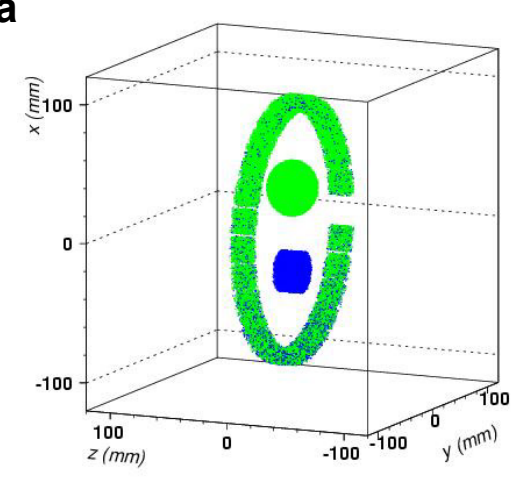

b

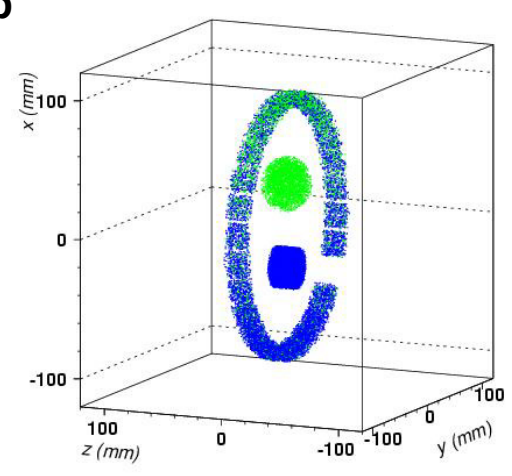

C

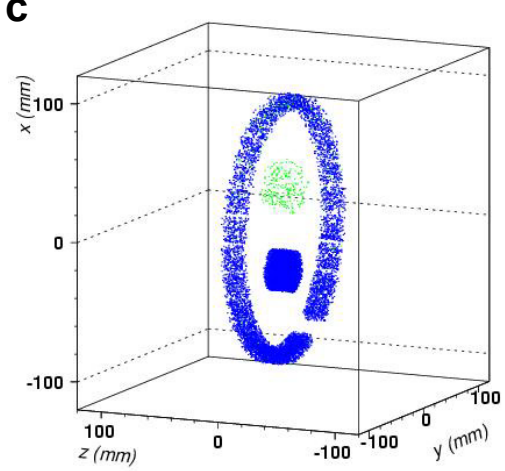

Fig. 1. Distributions of counts registered for the two decaying sources throughout three time-frames : (a) 0-2 min, (b) 7-9 min (c) 14-16 min. On all timeframes, one detector has been hidden (gap in the detector ring) to show the scanner rotation steps.

To model time-dependent processes and to keep them synchronized, a central "virtual clock" object models the passing of time in the acquisition. The user may specify the full duration of one or more time-frames, and subdivide each timeframe into a number of independent time-steps corresponding to fixed positions of the detectors and of the sources. For each event, the decaying isotope and its decay time are computed from the activity and from the radiotracer half-lives to model Poisson emission statistics while describing a continuous time flow. Thus, the number of events decreases exponentially from time-step to time-step and inside each time-step according to the decay kinetics of each radioisotope. Movements or combinations of movements such as rotations, translations or helical movements can be scripted for each volume of the scene. The position of each motive volume is then automatically updated at the beginning of each time-step. By redefining the sources or confining them to motive elements of the geometry, moving sources may be modeled as well.

\section{RESULTS}

\section{A. Simulation of decaying sources}

We simulated the decay of a sphere filled with ${ }^{15} \mathrm{O}$ and of a cylinder filled with ${ }^{11} \mathrm{C}$ throughout three independent time frames (0-2 $\mathrm{min}, 7-9 \mathrm{~min}$ and 14-16 $\mathrm{min})$. The simulated scanner, a model of a MicroPET [3], was set static during each time frame then was rotated by $30^{\circ}$ between each frame and the next one.

Fig.1 shows the spatial distribution of decay and detection points from the ${ }^{15} \mathrm{O}$ sphere (in green) and from the ${ }^{11} \mathrm{C}$ cylinder (in blue) for the three time-frames. As was expected, ${ }^{15} \mathrm{O}$ decays and hits dominate the first two minutes (first frame), but they already are in minority on the 7-9 min time-frame and have become almost negligible on the last time-frame. Fig.2 plots the count rate curve for each source: as may be seen on these plots, the simulated decay rates are in full agreement with the exponential decay rate that was computed from their halflives.

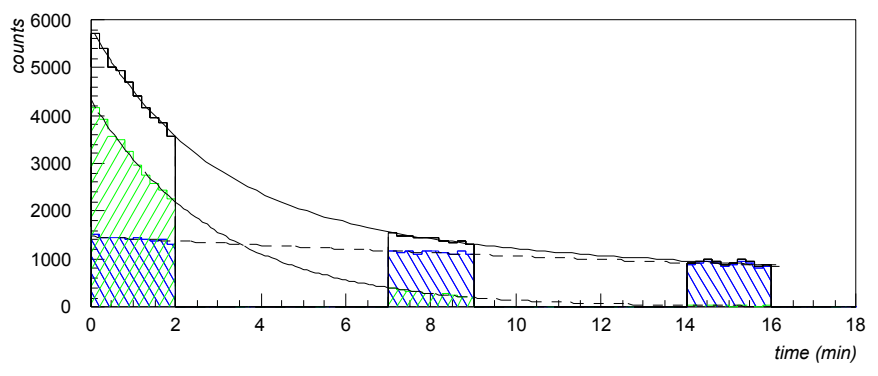

Fig. 2: Counts registered for the decay of sources of ${ }^{15} \mathrm{O}$ (rightward filling pattern) and ${ }^{11} \mathrm{C}$ (leftward pattern) throughout three time-frames (0-2, 7-9 and 14-16 $\mathrm{min}$ ). The dashed lines are theoretical decay curves from the isotope half-lives. The top curve shows the total number of counts from both sources.

\section{B. Simulation of count rate curves}

The precision of the event timing and random rates provided by GATE was assessed by simulating two independent beam sources of single $511-\mathrm{keV}$ gamma-rays impinging on two separate LSO crystals (Fig. 3). In order to reject autocoincidences on a crystal, a paralyzable dead-time equal to the coincidence window $w$ was applied to the detection of single events. As there were no gamma-pair emissions, all recorded coincidences were indeed random coincidences.

The simulated random rate $R$ was first compared to a simple model based on the single detection rate $S_{0}$ and on the single detection dead-time $w$ :

$R=2 w S_{0}^{2} e^{-2 w S_{0}}$

As shown by Fig. 3, the simulated rates (red squares) are in good agreement with the simple model (blue curve) at low activity, while small discrepancies appear $(<5 \%)$ at higher activities. This discrepancy disappears (black curve) when the loss of randoms due to multiples is taken into account as an additional paralyzable dead-time contribution:

$R=2 w S_{0}^{2} e^{-2 w S_{0}} e^{-w S_{0} e^{-w S_{0}}}$

Taking into account event timing at the simulation level allows the direct and accurate evaluation of count rate and 
sensitivity curves. This possibility is exemplified in Fig. 4, where we simulated acquisition rates for a point-source at the centre of an LSO-based small-animal PET scanner. Each sector was modeled with a paralyzable (Fig. 4a) or non-paralyzable (Fig. 4b) dead time of $8 \mu \mathrm{s}$ and a coincidence time window of $50 \mathrm{~ns}$. The plots show the rates of prompts $(P)$, randoms $(R)$ and true coincidences $(T)$. The Noise Equivalent Count (NEC) was computed as $N E C=T^{2} /(T+2 R)$.

\section{Simulation of rotating PET scanners}

Figs 5 and 6 plot simulations of the tomographic and projective resolutions for a MicroPET scanner set in rotation ( $1 \mathrm{deg} / \mathrm{s})$. For the tomographic resolutions, a point-like source ( $\varnothing=0.5 \mathrm{~mm}$ ) was moved by steps of $1 \mathrm{~mm}$ through the fieldof-view. For each source position, images were reconstructed using the 3DRP algorithm implementation from the STIR library [4], and resolution estimates were derived from Gaussian fits of profiles extracted from these images. For the projective resolution plot, a series of 100 simulations were performed with 6 point-sources located $10 \mathrm{~mm}$ apart and moving by steps of $100 \mu \mathrm{m}$. Sensitivity profiles were then computed for each sinogram bin and fitted with Gaussian

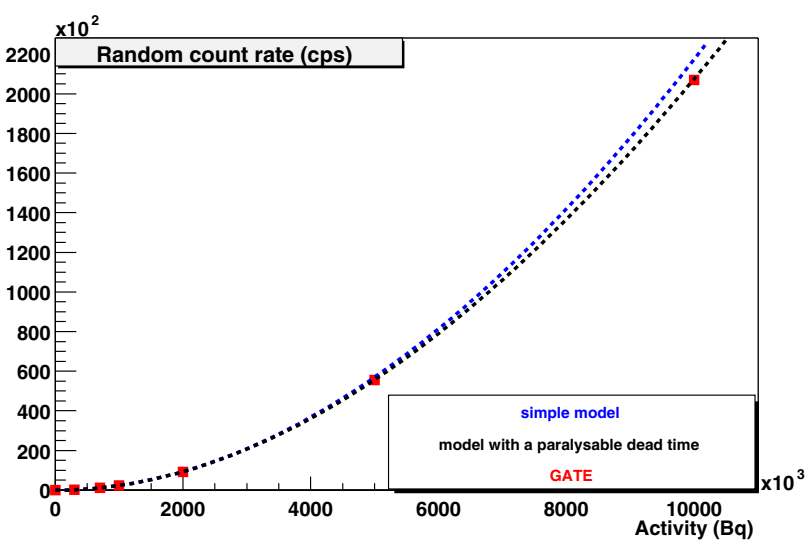

Fig. 3: Simulated random count rates (squares) versus theoretical predictions (curves) in a set-up with two independent gamma-ray sources.

function. For these two series of simulations, all gamma-ray interactions were taken into account, but positron range was not considered. Tomographic resolutions agree well with the data published for this scanner in the literature [5]. More difference is found for the projective resolution at the center: these discrepancies may be due to the neglecting of the
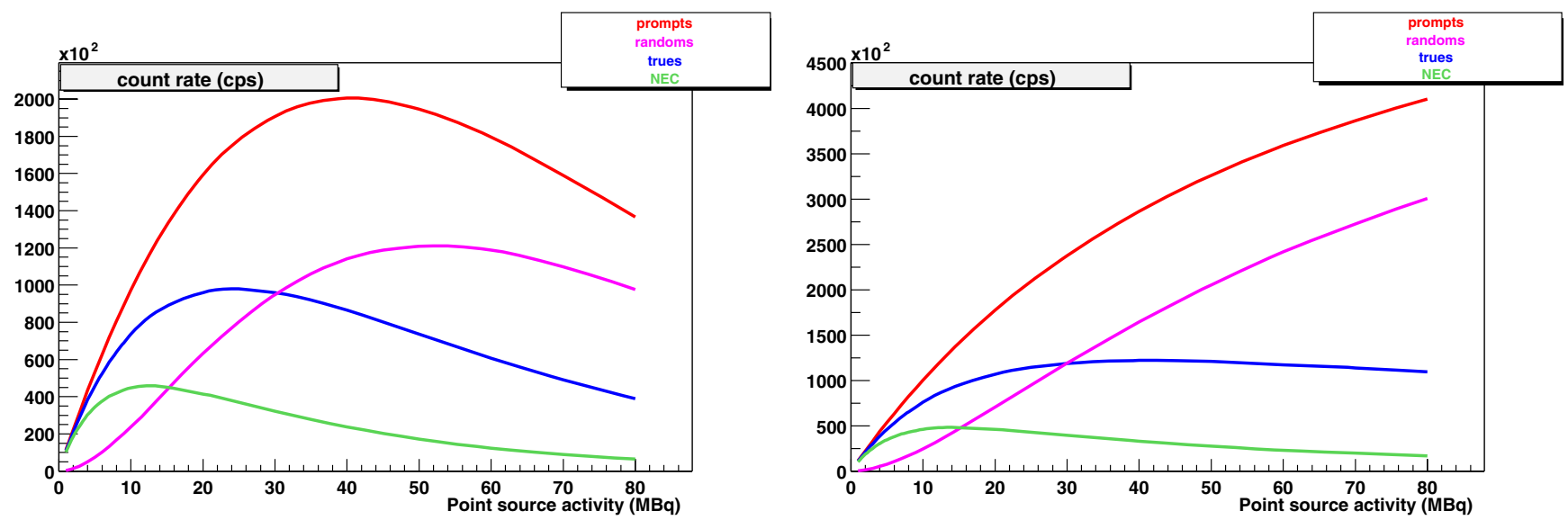

Fig. 4: Simulated count rates and NEC curves for a small-animal PET scanner and two dead-time models: paralyzable (left) and non-paralyzable (right).

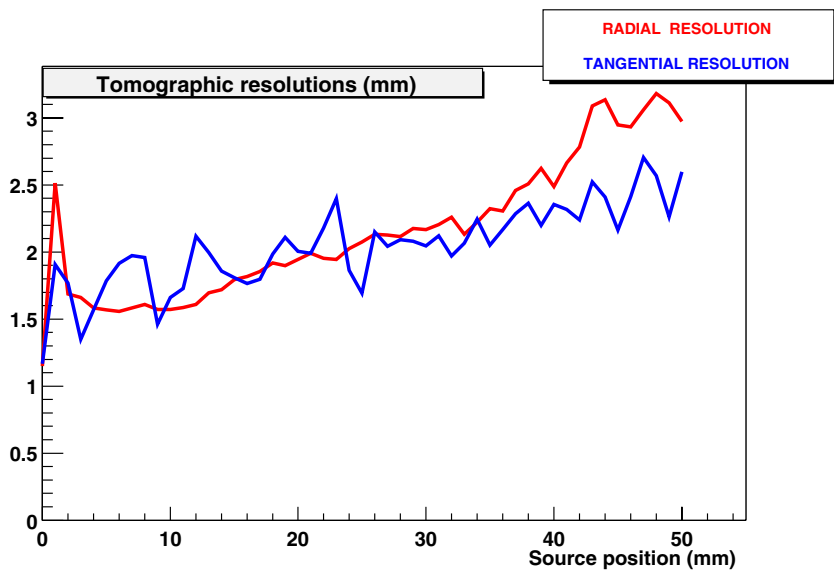

Fig. 5: Tomographic resolutions simulated for a rotating MicroPET scanner.

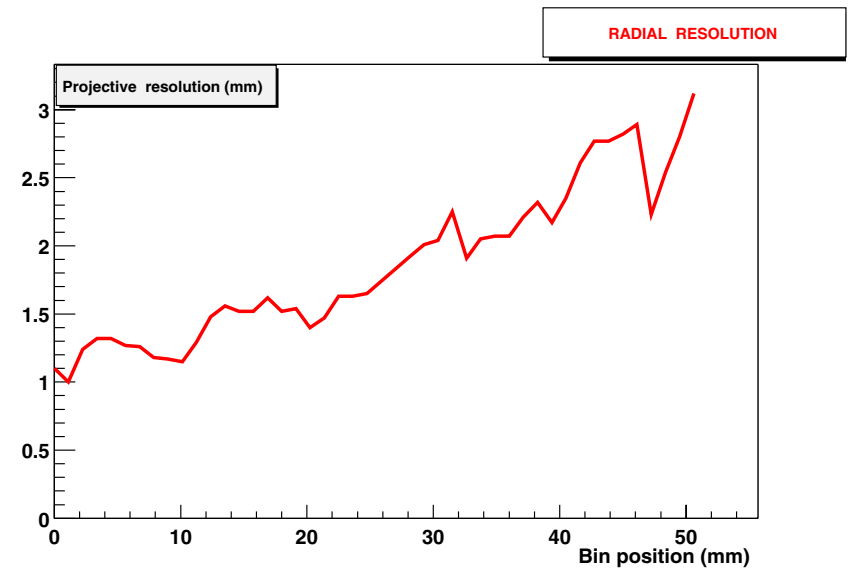

Fig. 6: Projective resolution simulated for a rotating MicroPET scanner. 
positron range (published measures were performed using ${ }^{22} \mathrm{Na}$ ), which significantly contributes to the intrinsic resolution of small-animal PET scanners.

Fig. 7 demonstrates the influence of scanner rotations for PET scanners with large inter-block gaps. In this case, a scanner with 16 dual-layer $8 \times 8$ crystal arrays of $2 \times 2 \times 8 \mathrm{~mm}^{2}$ crystals arranged in a ring geometry with $50 \mathrm{~cm}$ radius is described. In this simulation, two acquisitions of a multicylinder phantom were performed. On the static acquisition, a diamond-like pattern of low sensitivity lines may be seen on
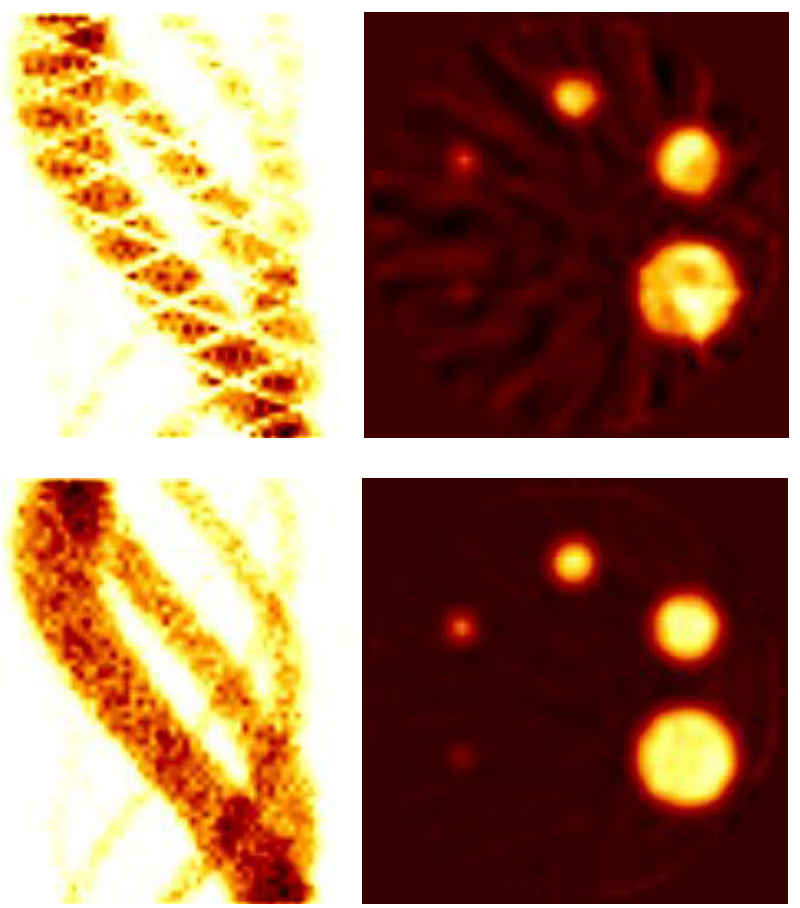

Fig. 7: Sinogram and reconstructed image for a PET scanner with large interblock gaps. (top) Static acquisition (bottom) Acquisition for a scanner in rotation. Diameters of the rod sources are 1, 2, 4, 7, 11, and $16 \mathrm{~mm}$.

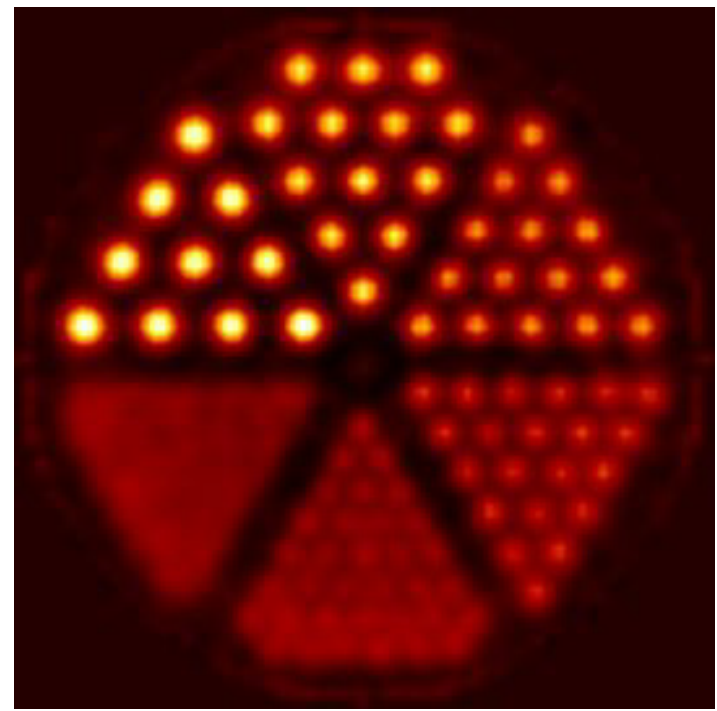

Fig. 8: Reconstructed image of a Derenzo-like phantom. Diameters of the rod sources are $1.5,2,2.5,3,3.5$, and $4 \mathrm{~mm}$. the sinogram, which result in poor homogeneity of the structures on the reconstructed image. By setting the scanner in rotation, the homogeneity of the sinogram and of the final image is restored.

These good results are exemplified on Fig. 8, where an acquisition of a Derenzo-like phantom for the same scanner design was simulated when set in rotation. Note that, for this image, neither the Compton scatter nor the positron range was taken into account, so that the final scanner resolution is significantly better than it would be in reality.

\section{Validation against a small-animal gamma-camera}

GATE was used to model a gamma camera prototype for small animal imaging tested at IASA (Institute of Accelerating Systems and Applications of Athens) [6]. It is made of a 3mmthick $\mathrm{CsI}(\mathrm{Tl})$ crystal array of $41 \times 41$ orthogonally arranged pillars, coupled to a PSPMT (R2486) manufactured by Hamamatsu. The gamma camera head is equipped with a LEHR parallel-hole collimator. Direct access to the anode wire signals is provided, allowing to compute the photon interaction position and its energy and to compare them to simulated values. The whole detection chain was modeled, including the physical interactions in the LEHR collimator, in the crystal pixels and in the PSPMT. The intrinsic performances of the
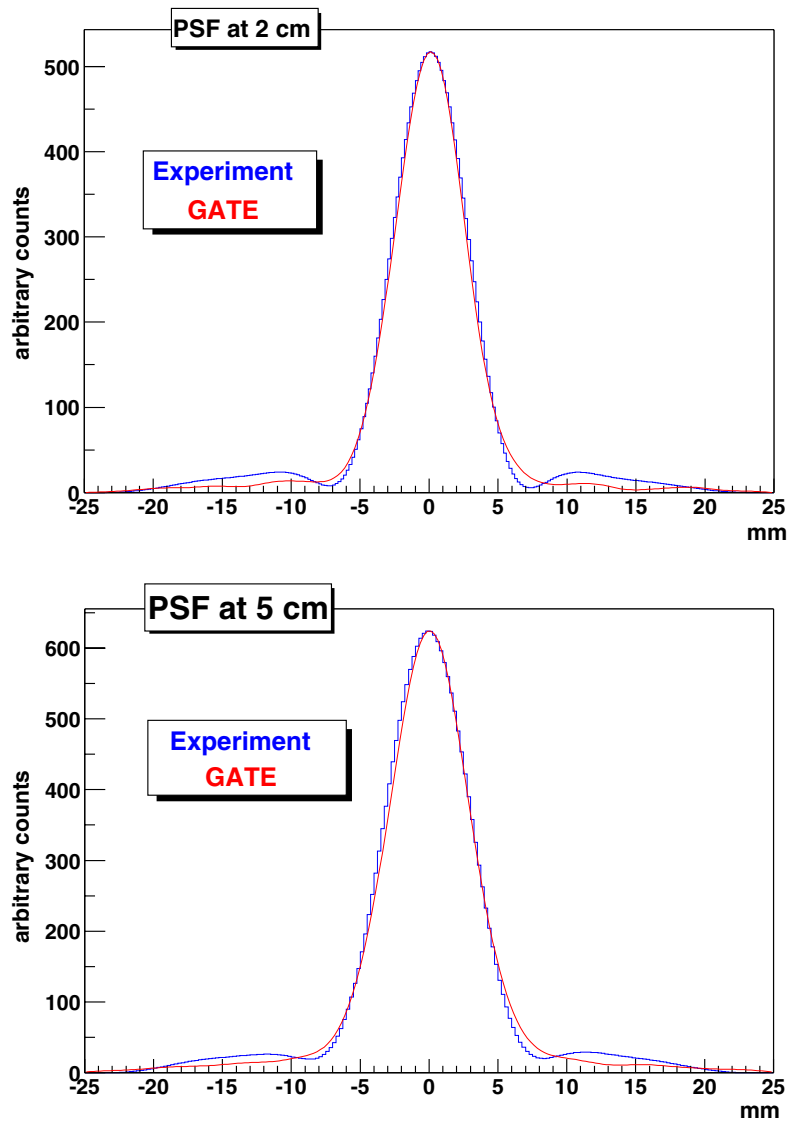

Fig. 9: Comparison of the simulated PSF vs experimental results for a source $2 \mathrm{~cm}$ (top) or $5 \mathrm{~cm}$ (bottom) away from the gamma camera 
prototype, as well as the intrinsic spatial resolution and the intrinsic energy resolution, were determined by measurements and used in the simulation to reproduce the intrinsic detection response of the gamma camera.

The basic performances of the gamma camera (point spread functions and energy spectra) in planar scintigraphic imaging were measured on the prototype in several configurations and then compared to the results given by the simulation. $\mathrm{A}^{99 \mathrm{~m}} \mathrm{Tc}$ point-like source (1.3 mm external diameter, $2 \mathrm{~mm}$, length) was placed at two positions ( 2 and $5 \mathrm{~cm}$ away from the camera) on the camera axis. The PSF and the energy spectra were measured experimentally in these configurations. 5 million photon histories were simulated in the angle of acceptance of the gamma camera: the simulated PSF for both positions and the energy spectrum for the source at $2 \mathrm{~cm}$ were compared to the experimental measurements.

The experimental and calculated PSF are compared on Fig. 9. A very good agreement was found for the simulation of the FWHM, with difference of less than $100 \mu \mathrm{m}$ between the simulated and the experimental values. Small discrepancies can be noticed in the tails of the PSF, which can be attributed to an experimental problem of charge collection on the wires.

Particular attention was paid to the simulation of the energy spectrum, and the backscatter from the PSPMT glass entrance window was found to be a major component: as the crystal is only $3 \mathrm{~mm}$ thick, about $30 \%$ of the $140 \mathrm{keV}$ incident photons leave the crystal without interacting and may scatter on the PSPMT glass entrance window. This backscatter was taken into account in the simulation by modeling the PSPMT as a single layer of material of appropriate dimensions and scattering properties. When this contribution was taken into account, the energy spectrum obtained with GATE matched well with the experimental one, as may be seen on Fig. 10.

\section{CONCLUSION}

We have presented the implementation of a Geant4-based PET simulation platform, designed to integrate time as a fundamental simulation parameter. We have shown results obtained for various time-dependent situations, including decaying sources, rotating scanners and plots of count rate versus field-of-view activity. We have also shown preliminary results of a validation on a gamma camera prototype, which gave excellent agreement between simulated and experimental

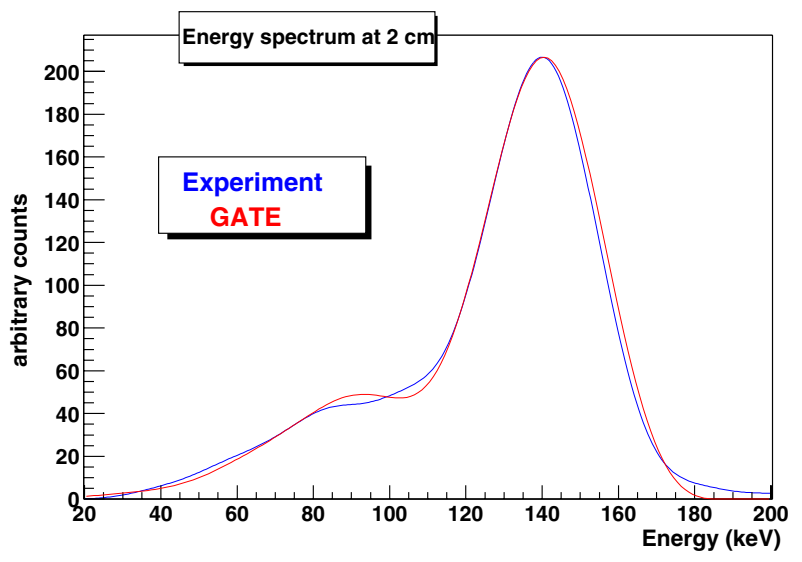

Fig. 10: Comparison of the simulated energy spectrum vs experimental results for a source $2 \mathrm{~cm}$ away from the gamma camera

data. These results illustrate the large range of situations that can already be realistically modeled.

Further development and validation of GATE is presently carried on within the OpenGATE Collaboration [7], with the objective to provide the academic community with a free software general purpose and versatile Geant4-based simulation platform for emission tomography. The "gridification" of GATE in currently under development and a public release of the software should be announced during summer 2003.

\section{REFERENCES}

[1] I. Buvat and I. Castiglioni, "Monte Carlo simulations in SPET and PET," Quaterly J. of Nucl. Med. 2002;46:48-61

[2] GEANT4 Collaboration, "GEANT4: A simulation toolkit," SLAC Report SLAC-PUB-9350, Aug. 2002

[3] S. R. Cherry, Y. Shao, R. W. Silverman, K. Meadors, S. Siegel, A. Chatziioannou et al., "MicroPET: a high resolution PET scanner for imaging small animals," IEEE Trans. Nucl. Sci 1997;44:1161-1166

[4] http://stir.irsl.org

[5] A. F. Chatziioannou, S. R. Cherry, Y. Shao, R. W. Silverman, K. Meadors, T. H. Farquhar et al., "Performance evaluation of microPET: a high-resolution lutetium oxyorthosilicate PET scanne for animal imaging," J. of Nucl. Med. 1999;40:1164-1175

[6] G. K. Loudos, K. S. Nikita, N. D. Giokaris, E. Styliaris, S. C. Archimandritis, D. Varvarigou et al., "A 3D high-resolution gamma camera for radiopharmaceutical studies with small animals," to be submitted

[7] http://www-iphe.unil.ch/ PET/research/gate/ 